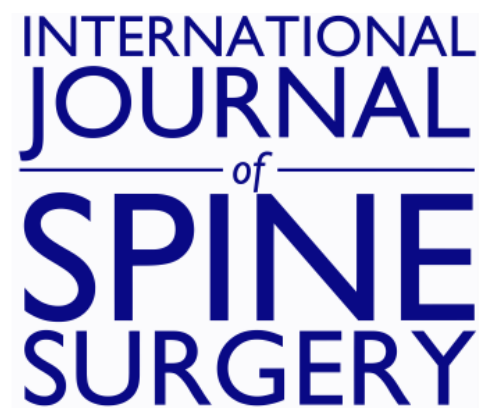

\title{
Intradiscal Delivery of Anabolic Growth Factors and a Metalloproteinase Inhibitor in a Rabbit Acute Lumbar Disc Injury Model
}

SAPAN D. GANDHI, TRISTAN MAERZ, SEAN MITCHELL, CASEY BACHISON, DANIEL K. PARK, JEFFREY S. FISCHGRUND and KEVIN C. BAKER

Int J Spine Surg 2020, 14 (4) 585-593

doi: https://doi.org/10.14444/7078

http://ijssurgery.com/content/14/4/585

This information is current as of April 26, 2023.

Email Alerts Receive free email-alerts when new articles cite this article. Sign up at: http://ijssurgery.com/alerts 


\title{
Intradiscal Delivery of Anabolic Growth Factors and a Metalloproteinase Inhibitor in a Rabbit Acute Lumbar Disc Injury Model
}

\author{
SAPAN D. GANDHI, MD,${ }^{1}$ TRISTAN MAERZ, PHD,${ }^{2}$ SEAN MITCHELL, MD,${ }^{1}$ CASEY BACHISON, MD ${ }^{1}$ \\ DANIEL K. PARK, MD,${ }^{1}$ JEFFREY S. FISCHGRUND, MD ${ }^{1}$ KEVIN C. BAKER, PHD ${ }^{1}$ \\ ${ }^{I}$ Department of Orthopaedic Surgery, Beaumont Health System, Royal Oak, Michigan, ${ }^{2}$ Department of Orthopaedic Surgery, University of Michigan, Ann Arbor, \\ Michigan
}

\begin{abstract}
Background: The purpose of our study was to examine the effect of controlled delivery of TGF- $\beta_{3}$, BMP-4, and TIMP-2 with a biocompatible biopolymer, chitosan, on an acutely injured intervertebral disc (IVD) in a rabbit model.

Methods: After conducting an in vitro analysis of the chondrogenic capacity of the biomolecule cocktail use (ie, TGF- $\beta_{3}$, BMP-4, and TIMP-2) and confirming stem cell viability in chitosan hydrogel, 15 New Zealand white rabbits underwent a lateral approach of the L1 to L4 IVDs. In each rabbit, the L2 to L3 IVD was left pristine, whereas the L1 to L2 and the L3 to L4 IVDs in each rabbit underwent nucleotomy via a 25-G needle, and the animal was subsequently randomized to no further treatment (defect only), chitosan alone, Chitosan + TGF- $\beta_{3}+$ BMP- 4 , or chitosan + TGF- $\beta_{3}+$ BMP-4 + TIMP-2. At 6 weeks after injury and intervention, the rabbits were killed and spines harvested to undergo quantitative T2 magnetic resonance imaging (MRI) and subsequent histologic analysis.

Results: In the in vitro analysis, cells treated with experimental media containing TGF- $\beta_{3}$, BMP-4, and TIMP-2 exhibited staining indicative of GAG production and began to exhibit a chondrocytic morphology. Quantitative T2 MRI mapping demonstrates that discs treated with chitosan, chitosan containing TGF- $\beta_{3}$ and BMP-4, or chitosan containing TGF- $\beta_{3}$, BMP-4, and TIMP-2 had consistently higher T2 relaxation times compared with defect-only discs. When the $\mathrm{T} 2$ relaxation times of each treatment group and defect-only discs were normalized to the healthy control disc, it was found that the T2 relaxation time of discs treated with chitosan containing TGF- $\beta_{3}$ and BMP-4 and discs treated with chitosan containing TGF- $\beta_{3}$, BMP-4, and TIMP-2 were significantly greater compared with defect-only discs $(P=$ .048 and $P=.013$, respectively). Histologically, animals that received chitosan only, or chitosan with TGF- $\beta_{3}$ and BMP4 , showed a significantly higher intensity of Safranin-O staining $(P=.016$ and $P=.02$, respectively) compared with control discs, whereas the difference in staining intensity in animals that received chitosan loaded with TGF- $\beta_{3}$, BMP-4, and TIMP-2 failed to achieve significance $(P=.161)$.
\end{abstract}

Conclusions: A combination of chitosan, TGF- $\beta_{3}$, and BMP-4 was effective at promoting regeneration in an acute disc injury rabbit model, whereas TIMP-2 did not have a significant effect.

Biologics

Keywords: disc degeneration, disc regeneration, metalloproteinase inhibitor, TGF-beta, bmp-4; disc injury

\section{INTRODUCTION}

The intervertebral disc (IVD) is a complex tissue that serves an important purpose in the biomechanical stability and functionality of the axial skeleton. The disc comprises a gelatinous core called the nucleus pulposus (NP), which is surrounded by the lamellar, fibrocartilagenous annulus fibrosus (AF). The end plates of the vertebral bodies form the inferior and superior boundaries of the disc. The cellular constituents of the NP possess a phenotype that is similar to chondrocytes within articular cartilage, whereas cells from the AF have been described as fibrochondrocytes. NP chondrocytes thrive in a gelatinous, 3-dimensional, amorphous environment, whereas AF fibrochondrocytes respond to the oriented, lamellar, nearly 2-dimensional structure provided by the concentric rings of the annulus. Because of these differences in cell phenotype and structure of the constituents of the disc, healing after injury has been a significant challenge.

Tissue engineering-based strategies, which rely on the use of scaffold materials, multipotent cells, and biomolecules, have been implemented in an attempt to regenerate the IVD. ${ }^{1,2}$ From a scaffold perspec- 
tive, hydrogel-based materials offer a promising biomimetic solution to regenerating NP tissue. Hydrogels are polymers that swell in response to various environmental stimuli, and many possess physical properties conducive to injection and subsequently facilitating the 3-dimensional proliferation of cells. Chitosan, which is a biopolymer based on the protein chitin found in crustacean shells, can be formulated to have gel-like properties. Chitosan and chitosan blends have been posited as materials that would be ideal for disc regeneration. ${ }^{3}$ The handling characteristics of the material can be easily tailored to suit clinical implementations (ie, injection). Additionally, the functional amine groups associated with chitosan interact with other biomolecules, which can be used to tailor drug delivery kinetics. We hypothesize that chitosan hydrogels can be used in the setting of IVD regeneration in terms of both providing an environment conducive to cell proliferation and growth, and serving as a vehicle for the in vivo delivery of cells and growth factors.

Although hydrogels may be the material of choice for scaffolds, the combination of molecular signals necessary for de novo disc tissue formation have not yet been fully elucidated. Our group has examined the effect of a combination of anabolic growth factors and an inhibitor of proteases on an in vitro culture of articular chondrocytes. ${ }^{4}$ Results indicated that a combination of transforming growth factor $\beta$ 3 (TGF- $\beta_{3}$ ), bone morphogenetic protein-4 (BMP4 ), and tissue inhibitor of matrix metalloproteinase2 (TIMP-2) was effective in increasing extracellular matrix production of these cells. Given the similarities in phenotype between articular chondrocytes and NP cells, an animal study was undertaken in an attempt to translate these results to a relevant animal model. The purpose of our study was to examine the effect of controlled delivery of TGF- $\beta_{3}$, BMP-4, and TIMP-2 with a biocompatible biopolymer, chitosan, on an acutely injured IVD in a rabbit model.

\section{MATERIALS AND METHODS}

\section{In Vitro Analysis of Chondrogenic Capacity of Biomolecule Cocktail}

Four mature New Zealand white rabbits were killed with pentorbital overdose. Bilateral femurs and tibias were harvested using gross dissection, and bone marrow was harvested from each intermedullary cavity. The bone marrow was immediately washed and immersed in basal medium (Dulbecco modified Eagle medium [DMEM], 10\% fetal bovine serum, $1 \% \mathrm{Pen} / \mathrm{Strep}$ ) on ice. An average of 10 to 12 $\mathrm{mL}$ of bone marrow was obtained per animal. A red blood cell lysis was performed using $30 \mathrm{~mL}$ of red blood cell lysis solution for 10 minutes at room temperature with frequent agitation. The resulting bone marrow cell solution was plated on T75 flasks to allow for the attachment of plastic-adherent cells. All flasks were washed 3 times with sterile phosphate-buffered saline after 24 hours to remove nonadherent cells, and adherent cells were visualized. This method has been previously shown to yield a population of multipotent cells able to maintain capacity of tri-lineage differentiation for multiple passages. ${ }^{5,6}$

Cells from the second passage (P2) exhibited a fibroblastic appearance and formed multiple highdensity fibroblastoid colonies in monolayer culture. P2 cells were plated at 25000 cells per well in a 24well plate. Chondrogenic differentiation was assessed in chondrogenic medium (DMEM, $1.25 \mathrm{mg} /$ $\mathrm{mL}$ bovine serum albumin, $1 \%$ fungizone, $1 \mathrm{mM}$ sodium pyruvate, $0.1 \mathrm{mM}$ ascorbic acid, $10^{-8} \mathrm{M}$ dexamethasone, and $10 \mathrm{ng} / \mathrm{mL}$ TGF- $\beta 3$ ). Furthermore, experimental media consisting of DMEM (with $1 \%$ fetal bovine serum and $1 \%$ fungizone) and supplemented with a combination of anabolic growth factors $\left(100 \mathrm{ng} / \mathrm{mL}\right.$ TGF- $\beta_{3}, 100 \mathrm{ng} / \mathrm{mL}$ BMP-4) and a catabolic inhibitor (100 ng/mL TIMP-2) was also assessed. These concentrations were selected based on our previous in vitro work that showed promising anabolic responses. ${ }^{4}$ Control cells were treated with basal medium. After exposure to the different media compositions, chondrogenic differentiation of isolated marrow cells was assessed by fixing the cells in 10\% neutral buffered formalin after 14 or 21 days in culture, and staining with $1 \%$ Alcian blue solution for 30 minutes. All wells were rinsed 3 times with $70 \%$ EtOH before being imaged using light microscopy (IX-71; Olympus Inc, Waltham, MA) to quantify staining intensity. All experiments were performed in $n=6$ wells per group per time point. Alcian blue staining intensities were calculated for each experimental well and compared to control cells.

\section{Synthesis of Chitosan Hydrogel and Effect on Stem Cell Viability}

In order to characterize the effect of the chitosan hydrogel on the viability of resident cells, cells 
Table 1. Treatment groups used in experimentation.

\begin{tabular}{llr}
\hline Group & \multicolumn{1}{c}{ Description } & Sample Size \\
\hline Chitosan & $15 \mu \mathrm{L}$ of chitosan hydrogel & 5 \\
Chitosan (TGF- $\beta_{3}+$ BMP-4) & $15 \mu \mathrm{L}$ of chitosan hydrogel loaded with $100 \mathrm{ng} / \mathrm{mL}$ each TGF- $\beta_{3}$ and BMP-4 \\
Chitosan (TGF- $\beta_{3}+$ BMP-4 + TIMP-2) & $15 \mu \mathrm{L}$ of chitosan hydrogel loaded with $100 \mathrm{ng} / \mathrm{mL}$ each TGF- $\beta_{3}$, BMP-4, and TIMP-2 & 5 \\
\hline
\end{tabular}

isolated from rabbit bone marrow were cultured in a chitosan hydrogel. The hydrogel consisted of $3 \mathrm{wt}$ $\%$ chitosan $(\mathrm{pH}=6.5)$, which was dissolved in $0.5 \%$ acetic acid at $4{ }^{\circ} \mathrm{C}$ for 48 hours. Dissolved chitosan was mixed with $0.025 \mathrm{~g} / \mathrm{mL}$ ammonium hydrogen phosphate. This resulted in physiologic $\mathrm{pH}$ equilibration $(\mathrm{pH}=7.4)$, cross-linking of chitosan molecules, and subsequent gelation into a viscous gel at $37^{\circ} \mathrm{C}$. At $4^{\circ} \mathrm{C}$, the hydrogel remained a viscous, free-flowing solution, which enabled the homogenous mixing of $10^{5}$ bone marrow cells per $1.0 \mathrm{~mL}$ of the cross-linked $\mathrm{Ch}$ solution, which was subsequently incubated at $37^{\circ} \mathrm{C}$ to allow for gelation. After 30 minutes, $1.5 \mathrm{~mL}$ of basal medium was added to each well containing the cross-linked chitosan/marrow cell mixture. New basal medium was replenished every third day. After 14 days of culture, media was aspirated from each well, and 1.5 $\mathrm{mL}$ of a live/dead staining solution was added to each well to quantify the fraction of viable cells. Following live/dead staining, 3-dimensional laser confocal microscopy was used to image a $250-\mu \mathrm{m}$ tall stack (5- $\mu \mathrm{m}$ step size) of each well to obtain a 3dimensional volume of stained cells within the chitosan matrix.

\section{Rabbit Nucleotomy Model and Treatment with Biomolecule-Loaded Chitosan Hydrogels}

Fifteen female New Zealand white rabbits (2.8$3.2 \mathrm{~kg}$ ) were used in accordance with a protocol approved by our Institutional Animal Use and Care Committee. Animals were anesthetized via intramuscular ketamine/xylazine, intubated, and maintained with inhaled isoflurane in a lateral position on the surgical table. After shaving a sterile prepping surgical site, a longitudinal midline incision was made over the lumbar spine, followed by a single fascial incision lateral to midline. Blunt dissection was employed to facilitate visualization of the IVDs at L1 to L2, L2 to L3, and L3 to L4, which were also confirmed with fluoroscopy. A $25-$ $\mathrm{G}$ spinal needle was then inserted into the nucleus of the L1 to L2 and the L3 to L4 discs, while keeping the L2 to L3 disc pristine. The center-center position of the needle within the nucleus was verified on multiple fluoroscopic views. Following confirmation of positioning, the disc was aspirated using a vacuum. The aspirated discs were then randomized to receive no further intervention (defect only), or treatment with chitosan, as shown in Table 1.

Hydrogels consisted of 3 wt \% chitosan crosslinked with ammonium hydrogen phosphate, and were synthesized as described for the previous injection. After cross-linking, biomolecules were homogenously mixed into the solution at a concentration of $100 \mathrm{ng} / \mathrm{mL}$ each. Hydrogels were maintained at $4^{\circ} \mathrm{C}$ to facilitate injection into the disc space through the inserted $25-\mathrm{G}$ needle. Treated discs received either chitosan alone, chitosan loaded with either TGF- $\beta 3$ and BMP-4, or chitosan loaded with TGF- $\beta 3$, BMP-4, and TIMP-2. Injection volumes remained consistent $(15 \mu \mathrm{L})$ regardless of the inclusion of biomolecules. Hydrogels were synthesized within 24 hours of injection.

After treatment of the discs, fascial and skin incisions were closed separately and animals were recovered from anesthesia. Animals were allowed ad libitum activity for a period of six weeks after surgery with free access to food and drink. At the sixth postoperative week, rabbits were anesthetized with intramuscular ketamine/xylazine and then administered an overdose of pentabarbitol. Lumbar spines were aseptically harvested and stored in zincbuffered $10 \%$ formalin until histologic processing.

\section{Quantitative T2 Magnetic Resonance Imaging}

Following necropsy and en bloc excision of the lumbar spine of each rabbit, quantitative T2 mapping was performed. This magnetic resonance imaging (MRI) modality has been previously shown to correlate to proteoglycan content of the nucleus pulposus and has been demonstrated to correlate to the degree of IVD degeneration. ${ }^{7,8}$ Lumbar spines were placed in a 6-channel send-receive wrist coil in a 3-T magnetic resonance imaging system. Following coronal and axial localizer sequences, multislice, multiecho T2-weighted maps were obtained. Coronal maps were obtained with $\mathrm{TR}=2000$, and $\mathrm{TE}=$ $11.1 \mathrm{~ms}, 22.2 \mathrm{~ms}, 33.3 \mathrm{~ms}, 44.4 \mathrm{~ms}$, and $55.5 \mathrm{~ms}$, with a $2-\mathrm{mm}$ slice thickness (0.2-mm slice gap), $180^{\circ}$ 

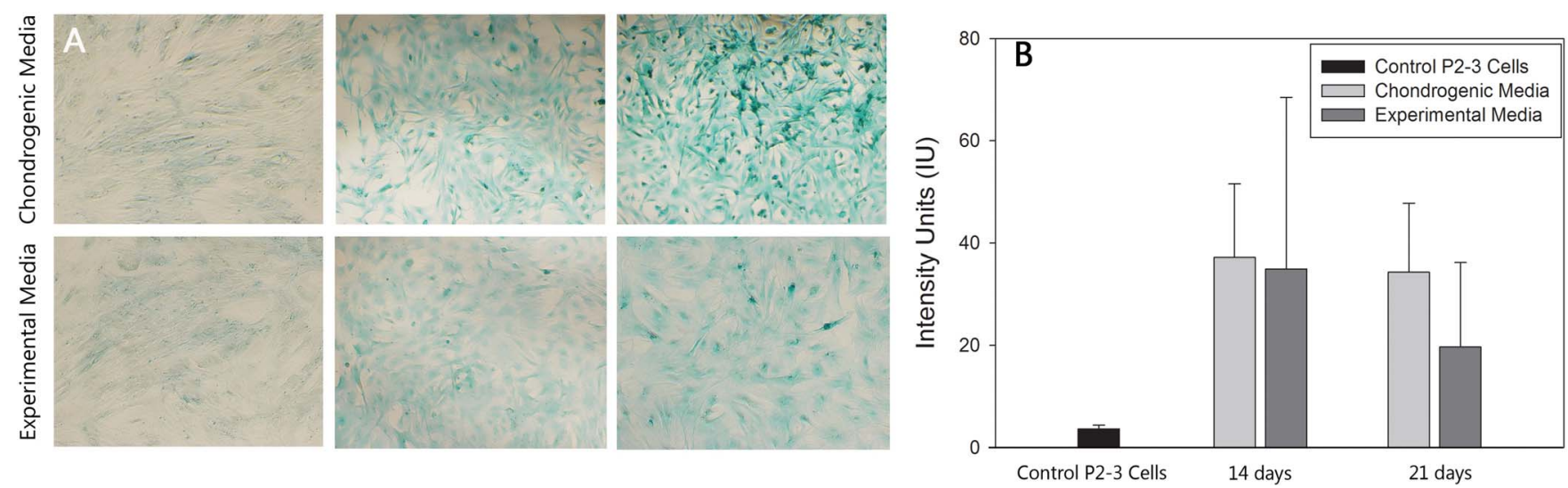

Figure 1. Quantitative results of differentiation assays. (A) Both chondrogenic media and experimental media containing TGF- $\beta_{3}$, BMP-4, and TIMP-2 induced timedependent increases in Alcian blue staining. (B) There was significantly greater staining intensity compared with control P2-3 cells treated with basal media.

flip angle, and 6 averages in a $140 \times 50$ pixel matrix (signal-to-noise ratio $[\mathrm{SNR}]=1.0)$ Axial maps of each disc were obtained with $\mathrm{TR}=1500$, and $\mathrm{TE}=$ $12.2 \mathrm{~ms}, 24.4 \mathrm{~ms}, 36.6 \mathrm{~ms}, 48.8 \mathrm{~ms}$, and $61.0 \mathrm{~ms}$, with a $2-\mathrm{mm}$ slice thickness (0.2-mm slice gap), $180^{\circ}$ flip angle, and 2 averages in a $102 \times 34$ pixel matrix $(\mathrm{SNR}=1.0)$. High-resolution T2 turbo spin-echo (TSE) sequences were acquired for gross visualization. T2 mapping was performed by curve fitting the logarithm of the signal intensity to echo time to obtain the quantitative $\mathrm{T} 2$ value in a pixel-by-pixel manner:

$$
S(\mathrm{TE})=S_{0} e^{-\mathrm{T} 2 / \mathrm{TE}}+C
$$

Parametric T2 maps were analyzed using region of interest processing. Axial maps of each disc (control, defect-only, and treatment) were used to quantify the mean T2 relaxation time of the NP and of the entire IVD. Analysis was performed in triplicate in a blinded fashion.

\section{Histologic Analysis}

Following MRI scans, the spines were then prepared for decalcified histology. The spines were kept intact during processing, such that the L1 to L2, L2 to L3, and L3 to L4 discs could be visualized simultaneously during microscopic characterization. Decalcified sagittal thin sections were stained with Safranin-O/Fast Green to aid in the quantification of proteoglycan content and distribution. Slides were digitized using an automated slide scanner at a magnification of $\times 20$. A custom-written MatLab algorithm was used to quantify Safranin-O staining intensity and total disc size. Three slides per animal, each containing an untreated nucleotomized, healthy, and treated nucleotomized disc, were also assessed by a blinded observer. The extent of IVD degeneration was qualitatively assessed using the grading scheme developed by Rutges et al. ${ }^{9}$

\section{RESULTS}

\section{In Vitro Bone Marrow Stromal Cell Differentiation}

Cells treated with differentiation media showed significantly greater staining intensity than control cells (Figure 1A). Furthermore, cells treated with experimental media containing TGF- $\beta_{3}$, BMP-4, and TIMP-2 exhibited staining indicative of GAG production and began to exhibit a chondrocytic morphology, suggesting chondrogenic differentiation due to treatment with the aforementioned growth factors and the proteinase inhibitor TIMP2. Quantitative image analysis of staining intensity demonstrated that cells treated with chondrogenic media exhibited significantly greater Alcian blue staining intensity, respectively (Figure 1B).

\section{Quantitative T2 MRI}

Quantitative T2 MRI mapping demonstrates that discs treated with chitosan, chitosan containing TGF- $\beta_{3}$ and BMP-4, or chitosan containing TGF$\beta_{3}$, BMP-4, and TIMP-2 consistently had consistently higher T2 relaxation times compared with defect-only discs. Furthermore, T2 mapping was sensitive to the increased proteoglycan content in the NP, because the mean T2 relaxation time was consistently higher in the NP compared with the whole disc.

When $\mathrm{T} 2$ relaxation time of each treatment group and defect-only discs were normalized to the healthy control disc, it was found that the whole-disc T2 relaxation times of discs treated with chitosan 


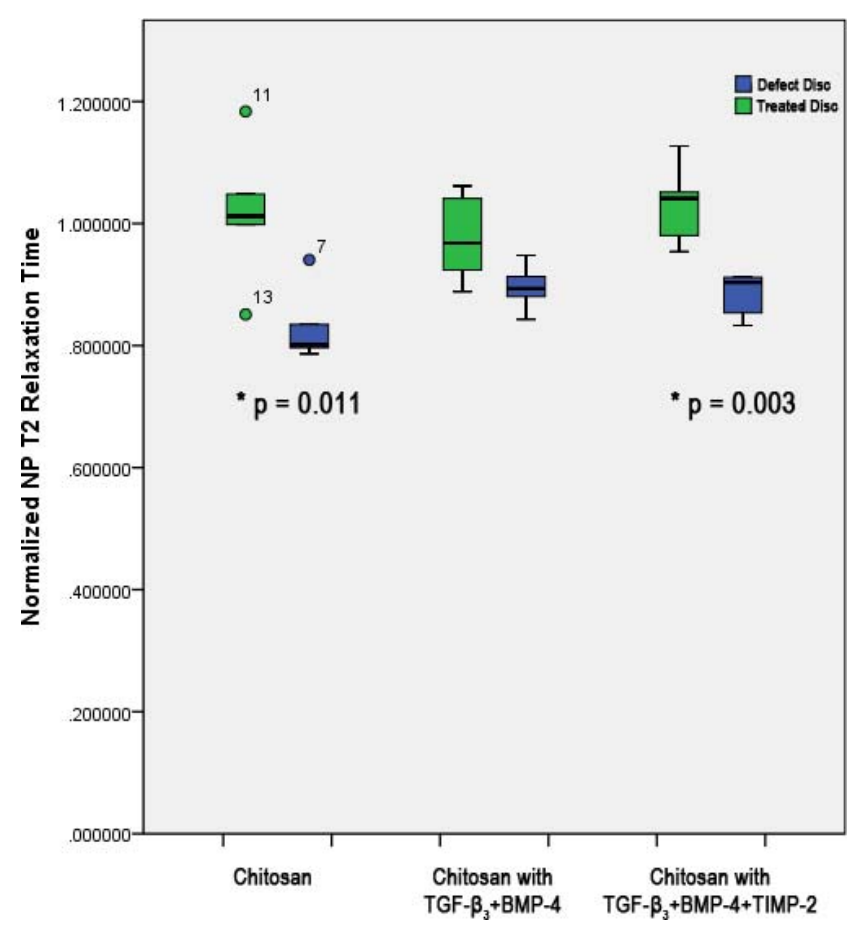

Figure 2. Normalized nucleus pulposus (NP)-only T2 relaxation times of defect-only discs, and discs treated with chitosan alone, chitosan containing TGF- $\beta_{3}$ and BMP-4, or chitosan containing TGF- $\beta_{3}$, BMP-4 and TIMP-2. ${ }^{*}$ Significant difference between treated and defect-only discs at $P<.05$.

containing TGF- $\beta_{3}$ and BMP-4 and discs treated with chitosan containing TGF- $\beta_{3}$, BMP-4, and TIMP-2 were significantly greater compared with defect-only discs $(P=.048$ and $P=.013$, respectively). Discs treated with chitosan only did not exhibit a difference in normalized $\mathrm{T} 2$ relaxation time compared with defect-only discs. When only the NP was analyzed, it was found that only discs treated with chitosan only and discs treated with chitosan containing TGF- $\beta_{3}$, BMP-4, and TIMP-2 exhibited greater normalized $\mathrm{T} 2$ relaxation time compared with defect-only discs $(P=.011$ and $P=$ .003 , respectively; Figure 2).

\section{Histologic Analysis}

Quantitative analysis of Safranin-O staining, which is proportional to sulfated glycosaminoglycan content, demonstrated higher intensity staining in all treated discs compared with those that received a defect without treatment (Figure 3A). Animals that received chitosan only or chitosan with TGF- $\beta_{3}$ and BMP-4 showed significantly higher intensity staining $(P=.016$ and $P=.02$, respectively) compared with their internal control defect discs, whereas the difference in staining intensity in animals that received chitosan loaded with TGF- $\beta_{3}$, BMP-4,
A

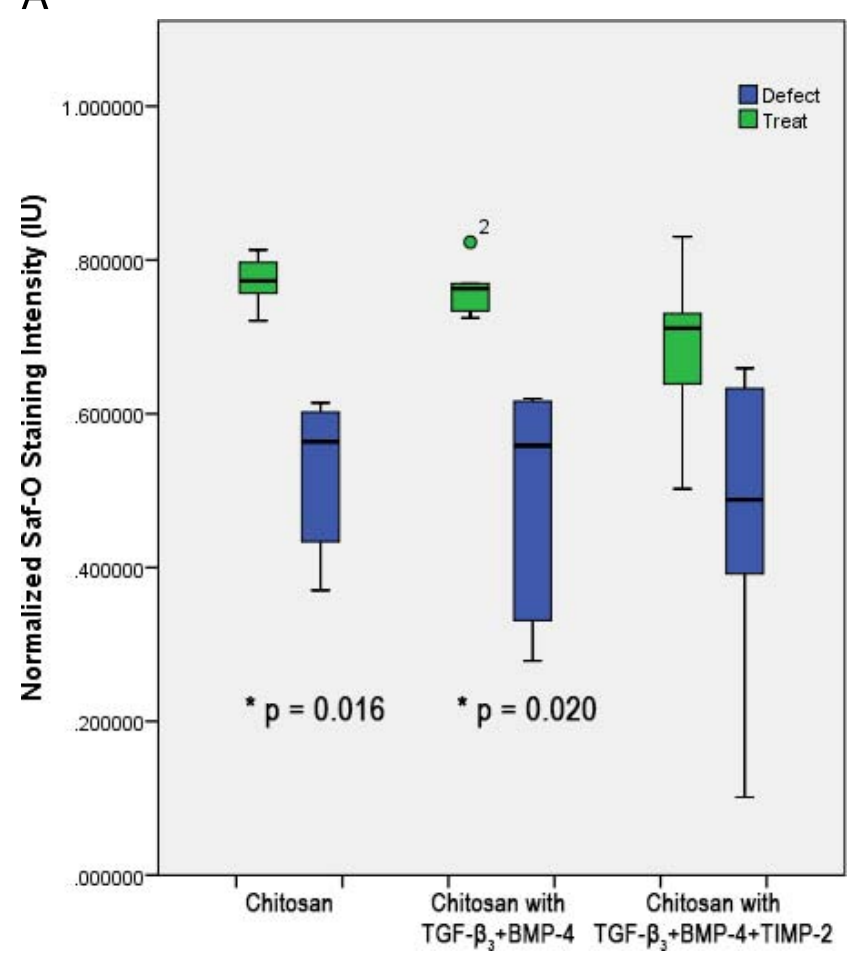

B

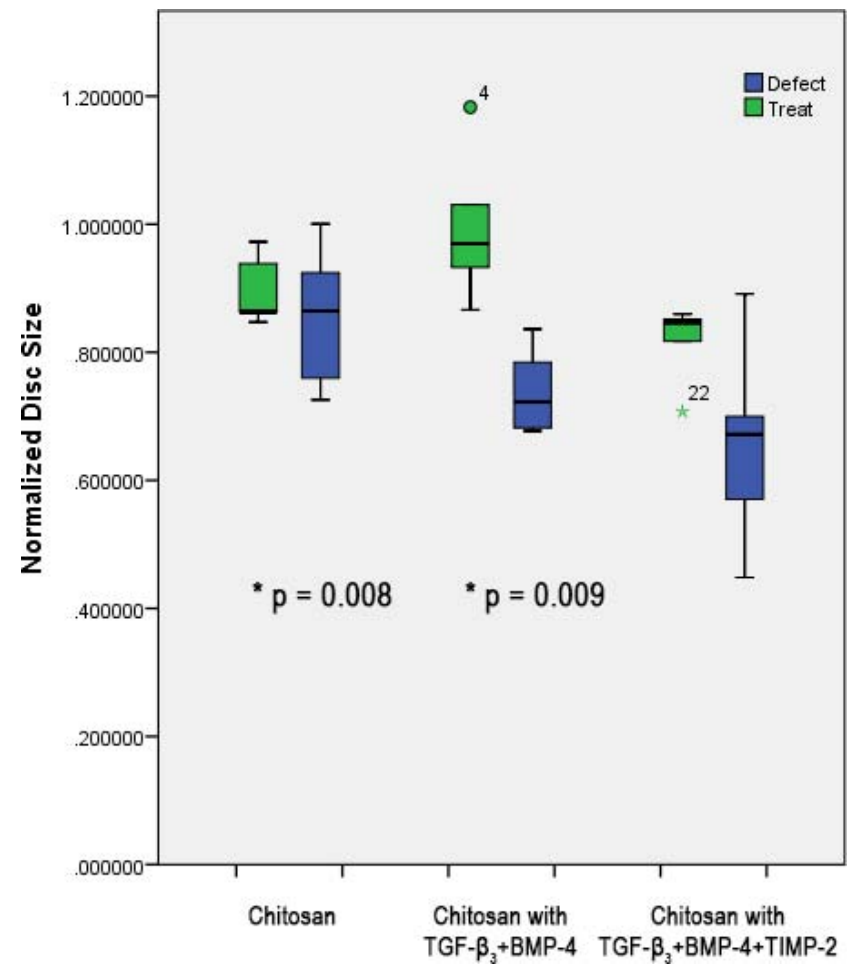

Figure 3. Quantitative analysis of Safranin-O staining intensity (A) and total disc size (B) from Safranin-O/Fast Green-stained decalcified sections. *Significant difference between treatment and defect discs. 
Table 2. Results of qualitative grading of Safranin-O/Fast Green slides according to the system developed by Rutges et al. ${ }^{9 a}$

\begin{tabular}{|c|c|c|c|c|c|c|c|}
\hline & End Plate & AF Morphology & AF/NP Boundary & NP Cellularity & NP Matrix & NP Matrix Staining & Total Score \\
\hline Healthy disc & $0.11 \pm 0.42$ & $0.32 \pm 0.55$ & $0.25 \pm 0.52$ & $0.64 \pm 0.62$ & $0.54 \pm 0.58$ & $0.04 \pm 0.19$ & $1.89 \pm 1.45$ \\
\hline \multicolumn{8}{|l|}{ Chitosan } \\
\hline Treatment $^{\mathrm{b}}$ & $0.14 \pm 0.53$ & $0.71 \pm 0.47$ & $1.00 \pm 0.88$ & $0.36 \pm 0.63$ & $0.43 \pm 0.51^{\mathrm{c}}$ & $0.00 \pm 0.00$ & $2.64 \pm 0.84$ \\
\hline Defect & $0.27 \pm 0.65$ & $0.82 \pm 0.87$ & $1.09 \pm 0.94$ & $0.45 \pm 0.82$ & $1.09 \pm 0.70$ & $0.55 \pm 0.93$ & $4.27 \pm 2.97$ \\
\hline \multicolumn{8}{|c|}{ Chitosan (TGF- $\beta_{3}+$ BMP-4) } \\
\hline Treatment & $0.00 \pm 0.00$ & $0.23 \pm 0.44$ & $1.08 \pm 0.64$ & $0.77 \pm 0.73$ & $0.54 \pm 0.52$ & $0.00 \pm 0.00$ & $2.62 \pm 1.26$ \\
\hline Defect & $0.08 \pm 0.28$ & $1.00 \pm 0.71$ & $1.38 \pm 0.51$ & $0.46 \pm 0.52$ & $1.00 \pm 0.71$ & $0.54 \pm 0.78$ & $4.46 \pm 2.73$ \\
\hline \multicolumn{8}{|c|}{ Chitosan (TGF- $\beta_{3}+$ BMP-4 + TIMP-2) } \\
\hline Treatment & $0.50 \pm 0.67$ & $0.83 \pm 0.72$ & $1.33 \pm 0.49$ & $0.08 \pm 0.29$ & $0.50 \pm 0.80$ & $0.33 \pm 0.78$ & $3.58 \pm 2.97$ \\
\hline Defect & $0.09 \pm 0.30$ & $0.09 \pm 0.83$ & $1.45 \pm 0.69$ & $0.27 \pm 0.47$ & $0.64 \pm 0.92$ & $0.54 \pm 0.93$ & $3.91 \pm 2.74$ \\
\hline
\end{tabular}

Abbreviations: AF, annulus fibrosus; NP, nucleus pulposus.

${ }^{a}$ Reference values for healthy discs are shown in the first row.

${ }^{\mathrm{b}}$ Statistical comparisons were made between the treatment and defect-only discs in each group using a Mann-Whitney $U$ test.

${ }^{\mathrm{c}}$ Bold formatting indicates statistical significance $(P<0.05)$.

and TIMP-2 failed to achieve significance $(P=.161)$. Semiautomated analysis of total disc area (Figure 3B) also demonstrated that discs treated with chitosan or chitosan with TGF- $\beta_{3}$ and BMP-4 were significantly larger than their respective internal control defect-only discs $(P=.008$ and $P=.009$, respectively).

Qualitative analysis of histologic sections using the Rutges classification ${ }^{9}$ showed that a healthy disc had an average total score of $1.89 \pm 1.45$, whereas the average total score for defect-only discs ranged from $3.91 \pm 2.74$ to $4.46 \pm 2.73$ (Table 2). Although average total scores for each of the treatment discs were lower than their respective defect-only discs, none achieved a statistically significant difference. Discs treated with chitosan alone achieved a significantly better score on nucleus pulposus matrix organization $(P=.02)$ and nucleus matrix staining $(P=.046)$ compared with the internal defect-only discs. Animals that had discs treated with chitosan loaded with TGF- $\beta_{3}$ and BMP-4 displayed significantly better annulus fibrosus organization $(P=$ $.005)$ and nucleus matrix staining $(P=.017)$ compared with defect discs.

No adverse or allergic reaction was noted by gross examination, MRI, or histology.

\section{DISCUSSION}

In general, degenerative disc disease is considered to be progressive and irreversible. Given its prevalence in the general population and its role as a public health concern, the regeneration of the IVD is of high interest among scientists and clinicians. However, it remains a lofty goal in which there are many technical challenges. In theory, attempts at a solution to this challenge combine a scaffold, growth factors, and multipotent cells to create the structural, signaling, and biologic environment for IVD regeneration. In our study, we examined the use of anabolic growth factors (TGF- $\beta_{3}$ and BMP4) and a protease inhibitor (TIMP-2), in combination with a chitosan scaffold-based delivery mechanism in the setting of a rabbit acute lumbar disc injury model. We found that, compared with injured discs without intervention, TGF- $\beta_{3}$ and BMP- 4 with a chitosan scaffold, as well as TGF- $\beta_{3}$, BMP-4, and TIMP-2 with a chitosan scaffold, were able to limit progression of proteoglycan loss and imaging characteristics of IVD degeneration. Histologically, a chitosan scaffold along with TGF- $\beta_{3}$ and BMP-4 displayed significantly better annulus fibrosis organization and nucleus matrix staining.

Our study is limited by our use of an acute disc injury model, as opposed to a more chronic model of IVD degeneration. Disc injury was created at the time of injection, which creates a reliable and reproducible model by which the effects of our intervention could be examined. However, an acute model has limited clinical translation, because most patients will present to the treating clinician after a significant amount of disc space loss, inflammation, and proteoglycan loss has already occurred in the IVD. However, for the purposes of initial evaluation of the effect our scaffold and growth factor combination, an acute model provided a reliable and reproducible method to examine its effects. Additionally, our exploratory study was limited by low sample size. Future studies should focus on higher group sizes to increase statistical power.

The search for the ideal scaffold to restore the biomechanical properties of the NP, as well as provide a suitable local environment for IVD regeneration, is ongoing. In our study we used chitosan, a biopolymer that has shown promise as a carrier for IVD regeneration. There is evidence that 
oxidative stress and subsequent apoptosis are responsible for some portion of IVD degeneration. ${ }^{10}$ Chitosan may provide protection from this phenomenon for NP cells, making it a suitable carrier and supplement for regenerative therapies. ${ }^{11,12}$ In addition, an ideal carrier should provide a suitable environment for cellular growth and differentiation. Although outside the scope of our current study, bone marrow-derived stem cells have had positive effects in the treatment of degenerative disc disease in both preclinical and clinical settings, and as such, the scaffold used in IVD regenerative treatment must be able to provide a suitable environment for the growth and differentiation of these cells. ${ }^{13-15}$ Chitosan has shown the ability to provide an appropriate medium for mesenchymal stem cell differentiation. ${ }^{16}$ Chitosan-based nanoparticles may also assist in the reduction of inflammation associated with disc damage. ${ }^{17}$ Lastly, the scaffold used in regenerative therapies must exhibit the mechanical properties of the NP to assist in its physiologic function as a "shock-absorber." Showalter and colleagues ${ }^{18}$ have demonstrated favorable biomechanical properties of a chitosan-based hydrogel in a human cadaveric model. In addition, Gullbrand et $\mathrm{al}^{19}$ have shown favorable mechanical properties of chitosan-based hydrogel in a goat preclinical model.

In our study, when analyzing only the NP, we found that chitosan-only discs exhibited greater normalized $\mathrm{T} 2$ relaxation time compared with defect-only discs. This suggests that even introduction of the mechanical and microenvironment support by the chitosan hydrogel itself helps to prevent continued degeneration, possibly for the reasons described previously. Although chitosanbased hydrogels have shown promise, other options have been described and explored. Gellan gumbased hydrogels, self-assembling peptidic hydrogels, hyaluronic acid-based gels, and fibrin gels have all been used in preclinical models. ${ }^{20-22}$

Altered growth factors play an intimate role in the development of degenerative disc disease. As such, regenerative therapies for IVD degeneration must aim to correct these changes. Because these alterations are likely local changes and not necessarily systemic changes, correction of unfavorable growth factor abnormalities may need to be delivered directly to the disc. ${ }^{23}$

TGF- $\beta$ is a family of anabolic growth factors that plays an important role in differentiation of mesenchymal stem cells into a chondrocytic lineage. Specifically, TGF- $\beta_{3}$ has been used to induce IVDlike cells in an in vitro setting, as well as to support AF matrix formation. ${ }^{24,25}$ A combination of TGF$\beta_{3}$ and BMP-4 has been used to form cartilage tissue in an in vitro model. ${ }^{26}$ Our study was able to show increased $\mathrm{T} 2$ relaxation time in discs treated with TGF- $\beta_{3}$ and BMP-4 compared with defect-only discs, implying that these anabolic growth factors prevented degeneration to some degree. Histologically, this combination assisted with increasing annulus fibrosis organization.

Matrix metalloproteinase-2 (MMP-2) and TIMP2 sit on the see-saw of extracellular matrix degradation and protection via inhibition, respectively. ${ }^{27}$ MMP-2 has been shown to be overexpressed in the setting of disc injury. ${ }^{28}$ In patients with cervical disc herniation, MMP-2 levels are increased, whereas TIMP-2 levels are decreased. ${ }^{29}$ Our study found that the addition of TIMP-2 to a chitosan scaffold and anabolic growth factors were effective at reducing disc degeneration compared with a defect-only disc.

Chitosan alone, as well as chitosan plus anabolic growth factors, was effective at reducing degeneration after an acute disc injury in a rabbit model. We did not find that the addition of TIMP-2 was helpful at reducing degeneration in our study. Future studies should examine the effect of these interventions in a chronic disc disease model that more closely resembles human pathology. In addition, the addition of mesenchymal stem cells to the growth factor and scaffold interventions may provide a more complete regenerative approach, and should be the target of future studies

\section{ACKNOWLEDGMENT}

This manuscript is dedicated to the memory of Harry N. Herkowitz, MD, chairman of the Department of Orthopaedic Surgery at Beaumont Health from 1991 to 2013.

\section{REFERENCES}

1. Ghorbani M, Ai J, Nourani MR, et al. Injectable natural polymer compound for tissue engineering of intervertebral disc: in vitro study. Mater Sci Eng C Mater Biol Appl. 2017;80:502508.

2. Steffen F, Smolders LA, Roentgen AM, Bertolo A, Stoyanov J. Bone marrow-derived mesenchymal stem cells as autologous therapy in dogs with naturally occurring interver- 
tebral disc disease: feasibility, safety and preliminary results. Tissue Eng Part C Methods. 2017;23(11):643-651.

3. Cheng YH, Yang SH, Lin FH. Thermosensitive chitosangelatin-glycerol phosphate hydrogel as a controlled release system of ferulic acid for nucleus pulposus regeneration. Biomaterials. 2011;32(29):6953-6961.

4. Pifer MA, Kibuule LK, Maerz T, Studzinski DM, Baker $\mathrm{KC}$, Herkowitz HN. In vitro response of human chondrocytes to a combination of growth factors and a proteinase inhibitor. Orthopedics. 2012;35(1):35-42.

5. Chong AK, Ang AD, Goh JCH, et al. Bone marrowderived mesenchymal stem cells influence early tendon-healing in a rabbit achilles tendon model. J Bone Joint Surg Am. 2007;89(1):74-81.

6. Toh WS, Liu H, Heng C, Rufaihah AJ, Ye CP, Cao T. Combined effects of TGF $\beta 1$ and BMP2 in serum-free chondrogenic differentiation of mesenchymal stem cells induced hyaline-like cartilage formation. Growth Factors. 2005;23(4):313-321.

7. Watanabe A, Benneker LM, Boesch C, Watanabe T, Obata T, Anderson SE. Classification of intervertebral disk degeneration with axial T2 mapping. AJR Am J Roentgenol. 2007;189(4):936-942.

8. Welsch GH, Trattnig S, Paternostro-Sluga $\mathrm{T}$, et al. Parametric T2 and T2* mapping techniques to visualize intervertebral disc degeneration in patients with low back pain: initial results on the clinical use of 3.0 Tesla MRI. Skeletal Radiol. 2011;40(5):543-551.

9. Rutges JPHJ, Duit RA, Kummer JA, et al. A validated new histological classification for intervertebral disc degeneration. Osteoarthritis Cartilage. 2013. 21(12):2039-2047.

10. Kim KW, Ha K-Y, Lee J-S, Rhyu K-W, An HS, Woo Y$\mathrm{K}$. The apoptotic effects of oxidative stress and antiapoptotic effects of caspase inhibitors on rat notochordal cells. Spine (Phila Pa 1976). 2007;32(22):2443-2448.

11. Jia P, Yu L, Tao C, Dai G, Zhang Z, Liu S. Chitosan oligosaccharides protect nucleus pulposus cells from hydrogen peroxide-induced apoptosis in a rat experimental model. Biomed Pharmacother. 2017;93:807-815.

12. He B, Tao H, Liu S, Wei A. Protective effect of carboxymethylated chitosan on hydrogen peroxide-induced apoptosis in nucleus pulposus cells. Mol Med Rep. 2015;11(3):1629-1638.

13. Pereira CL, Teixeira GQ, Ribiero-Machado C, et al. Mesenchymal stem/stromal cells seeded on cartilaginous endplates promote intervertebral disc regeneration through extracellular matrix remodeling. Sci Rep. 2016;6:33836.

14. Elabd C, Centeno CJ, Schultz JR, Lutz G, Ichim T, Silva FJ. Intra-discal injection of autologous, hypoxic cultured bone marrow-derived mesenchymal stem cells in five patients with chronic lower back pain: a long-term safety and feasibility study. J Transl Med. 2016;14:253.

15. Wang SZ, Jin J-Y, Guo Y-D, et al. Intervertebral disc regeneration using platelet-rich plasma-containing bone marrow-derived mesenchymal stem cells: a preliminary investigation. Mol Med Rep. 2016;13(4):3475-3481.

16. Richardson SM, Hughes N, Hunt JA, Freemont AJ, Hoyland JA. Human mesenchymal stem cell differentiation to NP-like cells in chitosan-glycerophosphate hydrogels. Biomaterials. 2008;29(1):85-93.

17. Teixeira GQ, Pereira CL, Castro F, et al. Anti- inflammatory chitosan/poly-gamma-glutamic acid nanoparticles control inflammation while remodeling extracellular matrix in degenerated intervertebral disc. Acta Biomater. 2016;42:168179.

18. Showalter BL, Elliott DM, Chen W, Malhotra NR. Evaluation of an in situ gelable and injectable hydrogel treatment to preserve human disc mechanical function undergoing physiologic cyclic loading followed by hydrated recovery. J Biomech Eng. 2015;137(8):081008.

19. Gullbrand SE, Schaer TP, Agarwal P, et al. Translation of an injectable triple-interpenetrating-network hydrogel for intervertebral disc regeneration in a goat model. Acta Biomater. 2017;60:201-209.

20. Silva-Correia J, Miranda-Goncalves V, Salgado AJ, et al. Angiogenic potential of gellan-gum-based hydrogels for application in nucleus pulposus regeneration: in vivo study. Tissue Eng Part A. 2012;18(11-12):1203-1212.

21. Wang B, Wu Y, Shao Z, et al. Functionalized selfassembling peptide nanofiber hydrogel as a scaffold for rabbit nucleus pulposus cells. J Biomed Mater Res A. 2012;100(3):646653.

22. Henriksson HB, Hagman M, Horn M, Lindahl A, Brisby $\mathrm{H}$. Investigation of different cell types and gel carriers for cellbased intervertebral disc therapy, in vitro and in vivo studies. $J$ Tissue Eng Regen Med. 2012;6(9):738-747.

23. Kim HJ, Park J-B, Won H-Y, Chang H. Serum levels of TGF-beta1, TIMP-1 and TIMP-2 in patients with lumbar spinal stenosis and disc herniation. Asian Spine J. 2007;1(1):811.

24. Steck E, Bertram H, Abel R, Chen B, Winter A, Richter W. Induction of intervertebral disc-like cells from adult mesenchymal stem cells. Stem Cells. 2005;23(3):403-411.

25. Hegewald AA, Zouhair S, Endres M, et al. Towards biological anulus repair: TGF-beta3, FGF-2 and human serum support matrix formation by human anulus fibrosus cells. Tissue Cell. 2013;45(1):68-76.

26. Sekiya I, Larson BL, Vuoristo JT, Reger RL, Prockop DJ. Comparison of effect of BMP-2, -4 , and -6 on in vitro cartilage formation of human adult stem cells from bone marrow stroma. Cell Tissue Res. 2005;320(2):269-276.

27. Cawston TE. Metalloproteinase inhibitors and the prevention of connective tissue breakdown. Pharmacol Ther. 1996;70(3):163-182.

28. Rastogi A, Kim H, Twomey JD, Hsieh AH. MMP-2 mediates local degradation and remodeling of collagen by annulus fibrosus cells of the intervertebral disc. Arthritis Res Ther. 2013;15(2):R57.

29. Zhuang HM, Xu GT, Wen SF, Guo YY, Huang Q. Altered expression of metalloproteinase-2 and tissue inhibitor of metalloproteinase-2 in cervical disc herniation patients. Genet Mol Res. 2016;15(2). doi: 10.4238/gmr.15027594.

Disclosures and COI: This research was funded by the Department of Orthopaedic Surgery at Beaumont Health. The authors declare no conflicts of interest related to the subject matter of this manuscript. All authors approved and take responsibility for the content of the manuscript.

Corresponding Author: Sapan D. Gandhi, 
MD, Department of Orthopaedics, 3535 West 13 Mile Road, Suite 744, Royal Oak, MI 48073. Phone: (860) 798-6105; Email: sapandgandhi@gmail.com.

Published 28 August 2020
This manuscript is generously published free of charge by ISASS, the International Society for the Advancement of Spine Surgery. Copyright (C) 2020 ISASS. To see more or order reprints or permissions, see http://ijssurgery.com. 